\title{
Molecular Architecture of a Sensory Ending Using Correlative Fluorescence and Electron Microscopy
}

Shigeki Watanabe*, Grigorios Oikonomou**, Shai Shaham**, and Erik M. Jorgensen*

* Howard Hughes Medical Institute and Department of Biology, University of Utah, 257 South 1400 East Salt Lake City, UT 84112

** Laboratory of Developmental Genetics, the Rockefeller University, 1230 York Avenue, New York, NY 10065

Mapping the molecular architecture of a protein within a cell and associating the protein with an organelle is essential for understanding the function of a protein. Fluorescence microscopy has been widely used to localize proteins in cells. However, the diffraction limit of light limits this approach [1], since two molecules that are within $\sim 200 \mathrm{~nm}$ cannot be resolved. Several microscopy techniques have evolved to break this limit [2]. Photo-activated localization microscopy (PALM) [3] and related techniques (stochastic optical reconstruction microscopy, STORM [4]; fluorescence photoactivation localization microscopy, fPALM [5]) resolve two closely spaced molecules by stochastically activating one molecule at a time and subsequently mapping the centroid of each to reconstruct an image. Although emission from each molecule is diffraction limited, the computer constructs an image with subdiffraction resolution because each molecule is localized independently. However, PALM lacks subcellular context.

PALM allows one to precisely pinpoint the location of a single molecule in a cell. However, precise localization in a field of black is not useful. Where is that protein in the context of cellular structure such as the plasma membrane or other organelles? Recently, we developed a correlative fluorescence electron microscopy (fEM) technique that overlays the protein localization revealed by TIRF fluorescence nanoscopy on the ultrastructures obtained by electron microscopy [6]. By imaging serial sections in which fluorescence is preserved and by reconstructing the stack, threedimensional localization of proteins to particular structures can be revealed.

Here we mapped actin molecules in the amphid sheath glia cells of Caenorhabditis elegans using serial section fEM reconstruction. The cilia of the sensory neurons are extended through the amphid channel, but the channels are typically so narrow that diffraction-limited fluorescence from actin-mEos molecules in the glia cell would illuminate the entire glia cell as well as the channels where cilia pass through. We reconstructed the region using serial section fEM and found that actin molecules are localized in the amphid sheath glia cell surrounding the sensory cilia but not in the channels (FIG. 1). Moreover, actin in the amphid channel is tightly localized at the plasma membrane surrounding the channel where filamentous structures were previously observed. These images illustrate that molecules can be localized to organelles even in small and convoluted tissues.

\section{References}

1. Cox, G. \& Sheppard, C.J. Practical limits of resolution in confocal and non-linear microscopy. Microscopy Research and Technique 63, 18-22 (2004).

2. Hell, S.W. Far-field optical nanoscopy. Science 316, 1153-1158 (2007).

3. Betzig, E. et al. Imaging Intracellular Fluorescent Proteins at Nanometer Resolution. Science 313, 1642-1645 (2006).

4. Rust, M.J., Bates, M. \& Zhuang, X. Sub-diffraction-limit imaging by stochastic optical reconstruction microscopy (STORM). Nat. Methods 3, 793-795 (2006). 
5. Hess, S.T., Girirajan, T.P.K. \& Mason, M.D. Ultra-high resolution imaging by fluorescence photoactivation localization microscopy. Biophys. J 91, 4258-4272 (2006).

6. Watanabe, S. et al. Protein localization in electron micrographs using fluorescence nanoscopy. Nat. Methods 8, 80-84 (2011).

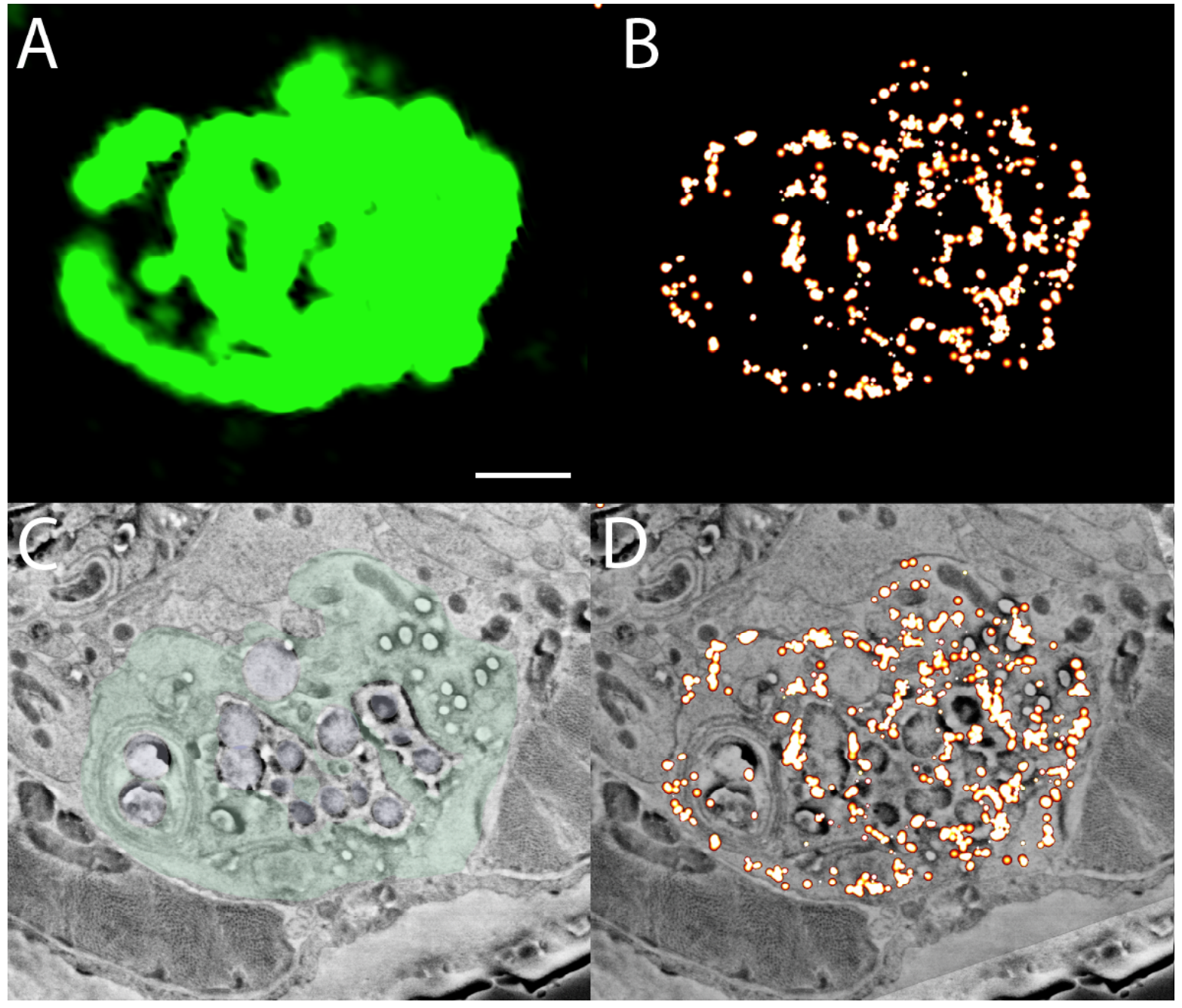

FIG. 1. Actin localization by correlative PALM-EM. Sum TIRF (A), PALM (B), and SEM (C) images of a ultrathin section $(\sim 70 \mathrm{~nm})$ from a transgenic animal expressing Actin-tdEos. Green shading indicates the dimensions of the sheath cell in C. (D) An overlay of (B) and (C) depicting actin localization in the sheath cell. Scale bar $=1 \mu \mathrm{m}$. 